\title{
Uso del caucho granulado en mezclas asfálticas: Una revisión literaria
}

\section{Use of granulated rubber in asphalt mixtures: A literary review}

\section{Luz Magali Hoyos Díaz}

Escuela Profesional de Ingeniería Civil

Facultad de Ingeniería Arquitectura y Urbanismo

Universidad Señor de Sipan

Perú

hdiazluzm@crece.uss.edu.pe

\section{Katyuska del Carmen Puicon Herrera}

Escuela Profesional de Ingeniería Civil

Facultad de Ingeniería Arquitectura y Urbanismo

Universidad Señor de Sipan

Perú

hepuiconka@crece.uss.edu.pe

\section{Sócrates Pedro Muñoz Pérez}

Director de la Escuela Profesional de Ingeniería Civil, Facultad de Ingeniería Arquitectura y Urbanismo Universidad Señor de Sipan

Perú

msocrates@crece.uss.edu.pe

Fecha de recepción: 29 de octubre de 2020 / Fecha de aprobación: 16 de febrero de 2021

\section{RESUMEN}

Cada año se producen millones de toneladas de neumáticos desechados, lo cual presenta un grave problema ambiental. Debido a esto, en la actualidad se está usando el caucho granulado (CR) en el diseño de mezclas asfálticas, ya que proporcionan propiedades fundamentales en ello, como la durabilidad y la resistencia. En la presente recopilación literaria se analizaron diferentes artículos con el objetivo de descifrar los procesos y métodos que se emplean con el uso del CR, identificar los porcentajes óptimos y la mejora que éste proporciona en las mezclas asfálticas. Se halló que los porcentajes óptimos de CR en las mezclas asfálticas varían en un rango de $1 \%-20 \%$ en peso de la mezcla total. Asimismo, utilizar el CR aporta diferentes beneficios a la mezcla tales como: resistencia a la tracción indirecta, a la susceptibilidad, a la humedad, a la formación de surcos, a la permeabilidad y al envejecimiento; aumenta la vida útil de las mezclas a la fatiga y disminuye el ruido. Se revisaron 50 artículos indexados en la base de datos Scopus entre los años 2006 y 2020 sobre el uso de CR en mezclas asfálticas, daño por humedad, resistencia a la formación de surcos, resistencia a la fatiga y resistencia a baja temperatura.

\section{ABSTRACT}

Millions of tons of discarded tires are produced each year, presenting a serious environmental problem. Due to this, granulated rubber (CR) is currently being used in the design of asphalt mixtures, since they provide fundamental properties in it, such as durability and resistance. In this research, different articles were analyzed with the aim of deciphering the processes and methods used with the use of CR, identifying the optimal percentages and the improvement it provides in asphalt mixtures. It was found that the optimum percentages of CR in the asphalt mixtures vary in a range of $1 \%-20 \%$ by weight of the total mixture. Likewise, using CR provides different benefits to the mixture such as: resistance to indirect traction, susceptibility, humidity, grooving, permeability, and aging; increases the useful life of the mixtures to fatigue; and reduces noise. Fifty articles indexed in the Scopus database between 2006 and 2020 were reviewed on the use of $C R$ in asphalt mixtures, moisture damage, resistance to furrow formation, resistance to fatigue and resistance to low temperature.

Keywords: Granulated rubber, moisture damage, dry process, wet process

Palabras clave: Caucho granulado, daño por humedad, proceso seco, proceso húmedo 


\section{INTRODUCCIÓN}

A nivel mundial, la gran mayoría de los neumáticos de desecho se depositan en vertederos, con consecuencias ecológicas catastróficas y, en particular, serias amenazas para la salud humana (por ejemplo, incendios, plagas y contaminación del suelo) (Rodríguez, Tarpoudi, Cavalli, Poulikakos y Bueno, 2020), debido a la creciente conciencia ambiental, y el afán por recuperar el caucho granulado (CR) y transformarlo en un material eficiente, por eso su uso ha ido aumentando en la producción de asfalto ya que varios investigadores han argumentado que el asfalto modificado con CR se comporta más eficazmente que el concreto asfáltico convencional (Wang y Zeng, 2006).

Según las experiencias obtenidas con el uso de CR en varios países mostraron el excelente comportamiento estructural y funcional de este tipo de material (Dantas, Farias, Pais y Pereira, 2006). La motivación más importante detrás de esta aplicación del CR fue la mejora del rendimiento del pavimento, incluida una mayor vida útil a través de la resistencia al agrietamiento y la formación de surcos, disminución del ruido del tráfico, reducción de los costos de mantenimiento y aumento de la seguridad del tráfico mediante la resistencia al deslizamiento y la reducción del rocío que daña la visión durante las inclemencias (Shen, Amirkhanian, Lee y Putman, 2006).

Existe una demanda considerable de uso de neumáticos de desecho en aplicaciones de ingeniería civil, que van a mitigar daños al medio ambiente, por eso no solo es una forma innovadora de reciclar neumáticos usados, sino también uno de los métodos más efectivos para extender la vida útil del pavimento. En consecuencia, los pavimentos construidos con mezclas de caucho asfáltico (AR) tienen muchos beneficios, como una durabilidad mejorada y una resistencia mejorada a la fatiga iniciada en la superficie y al agrietamiento por reflexión, resistencia mejorada al envejecimiento y a la oxidación debido a un mayor contenido de aglomerante (Bressette, Zhou, Stonex y Hicks, 2008).

La incorporación de CR de llantas de desecho en mezclas de pavimento asfáltico está diseñada para mejorar las propiedades del asfalto mediante la reducción de la susceptibilidad inherente a la temperatura del aglomerante (Kim, Lee y Amirkhanian, 2010), estas al ser modificadas con CR con más del 20\% de migajas tiene mayor viscosidad, mejor flexibilidad, propiedades antifatiga y antienvejecimiento mejoradas (Dong y Tan, 2011). Asimismo, el CR se incorpora a las mezclas asfálticas como una de las estrategias exitosas para mejorar la resistencia a la fatiga del pavimento asfáltico (Saeed, Aman, Ahmad, Yero y Chinade, 2018).

Se ha demostrado que la utilización de CR para producir AR para pavimentos de mezclas asfálticas es una forma económica y ambiental para la eliminación de llantas de desecho (Gong, et al., 2019). Por lo tanto, estos residuos pueden reducirse transformándolos en un material adecuado para añadir a las mezclas de pavimentos de carreteras este material adicional hecho de neumáticos usados se llama CR (Kartika, Hadiwardoyo y Sumabrata, 2019). En consecuencia, el modificador de CR es uno de los modificadores del asfalto más populares debido a los beneficios económicos y las propiedades físicas y reológicas deseadas de los ligantes de asfalto y mezclas asfálticas (Khalili, Jadidi, Karakouzian y Amirkhanian, 2019).

\section{DESARROLLOY DISCUSIÓN}

\section{Porcentaje óptimo de caucho granulado en mezclas asfálticas}

Cao (2007) realizó pruebas de laboratorio con tres tipos de mezclas asfálticas con adición de CR en diferentes contenidos de $(1 \%, 2 \%$ y $3 \%$ en peso de la mezcla total) y una mezcla convencional sin caucho, denominada mezcla-control, las muestras fueron comprimidas por 75 golpes por cara con el martillo Marshall estándar. A partir de los ensayos de Marshall y tracción indirecta realizados por el autor, determinó que el contenido óptimo de CR en la mezcla asfáltica de $3 \%$ tiene el mejor rendimiento tanto a alta temperatura $\left(60^{\circ} \mathrm{C}\right)$, como a baja temperatura $\left(10^{\circ} \mathrm{C}\right)$.

Hamzani y colaboradores elaboraron una investigación para determinar la resistencia a la compresión de pavimentos semiflexibles mediante el uso de CR como sustituto de asfalto. El asfalto fue reemplazado por 3\%, 4\% y 5\% de CR y se realizó la prueba Marshall. La prueba de resistencia a la compresión se realizó con base en ASTM D1074-02 a la edad de 14 días. Los resultados de la prueba mostraron que la mayor resistencia a la compresión se alcanza con el 5\% CR, que es de 15,43 $\mathrm{MPa}$ (Hamzani, Munirwansyah, Hasan y Sugiarto, 2019). Si bien es cierto la norma ASTM D1074-02 no menciona el tiempo en el que se debe de ejecutar el ensayo los autores Hamzani, Munirwansyah, Hasan y Sugiarto, tomaron el criterio de realizar la prueba a los 14 días. 
Ahmad y colaboradores realizaron un estudio con residuos de CR y baquelita para estudiar las propiedades mecánicas mediante una proporción de adición de 4,0\%, 8,0\%, 12,0\%, $16,0 \%$ y $20.0 \%$. Se diseñaron y probaron las mezclas Marshall para determinar la densidad, estabilidad Marshall, flujo, vacíos de aire y propiedades de rigidez para las muestras de control, CR baquelita. Concluyendo que la adición de CR y baquelita en un $12 \%$ mejora significativamente las propiedades de la mezcla de asfalto casi duplica la resistencia de estabilidad Marshall en comparación con la muestra de control (Ahmad, Beddu, Hussain, Manan y Itam, 2019).

Munir y colaboradores evaluaron las propiedades de los ligantes asfálticos modificados con $\mathrm{CR}$ reaccionado y activado en comparación con los ligantes asfálticos tradicionales que se utilizan en los EAU. Se diseñaron dos aglutinantes al $20 \%$ y al 30\% con CR en peso de asfalto. El trabajo de prueba de laboratorio concluyó que las simulaciones de desempeño del pavimento mostraron que el aglomerante modificado con el $20 \%$ de CR es el más prometedor para producir el pavimento de mejor desempeño con la menor cantidad de grietas por fatiga y surcos, también ofrece una solución rentable al extender la vida útil del pavimento en un promedio de 8 años con actividades mínimas de mantenimiento y rehabilitación (Munir, Elnour, Huda y Zeiada, 2020).

\section{Procesos principales para producir mezclas asfálticas modificadas con caucho granulado}

De acuerdo con la revisión bibliográfica, existen dos procesos más empleados para la producción del asfalto con la adición de CR, denominados: proceso húmedo, en el cual el CR modifica al asfalto y el proceso seco el CR es añadido como un porcentaje del agregado fino.

\section{Proceso caliente o húmedo}

El proceso húmedo original, inventado por Charles McDonald, conduce a un producto con una serie de beneficios que son básicamente todo relacionado con el aumento de elasticidad y viscosidad del asfalto a altas temperaturas que permite un mayor espesor de película en pavimento de mezclas sin drenaje excesivo o sangrado (Lo Presti, 2013).

Ma y colaboradores realizaron un estudio para evaluar los efectos de los aditivos de asfalto (denominados Sas y Evm) en la mezcla tibia, sobre la temperatura de compactación y las propiedades del asfalto y mezcla de asfalto modificado con $\mathrm{CR}$, mediante el proceso húmedo, en el que se añadió un porcentaje de $25 \%$ de CR en peso del asfalto base, se tuvo como resultado que los aditivos podrían reducir la temperatura de compactación de mezclas de asfalto con $\mathrm{CR}$ en $10^{\circ} \mathrm{C}$ a $20^{\circ} \mathrm{C}$. Sin embargo, tienen diferentes influencias sobre las propiedades reológicas del asfalto con CR y rendimiento de la mezcla con CR (Ma, Wang, Zhao, Huang y Wang, 2016).

En su investigación, Al-Salih (2020) tuvo como objeto resolver dos problemas principales: la eliminacion segura de los neumáticos desechados y estudiar el comportamiento de formación de surcos en mezclas asfalticas modificadas con $\mathrm{CR}$, mediante el proceso húmedo, en el que se añadio un $6 \%$ del peso del asfalto, obtuvo como resultados que las mezclas preparadas con asfalto modificada con CR muestran buena resistencia a la formación de surcos y el beneficio de este estudio es que los desechos de llantas se eliminan de una forma ecológica (Al-Salih, 2020).

\section{Proceso seco}

En su investigación, Hernández y colaboradores determinaron el porcentaje óptimo de adición de CR en la mezcla, mediante el proceso seco, en la cual el CR se obtuvo mediante el método de trituración, el tamaño de CR es de $0,45 \mathrm{~mm}$ que corresponde a un tamaño máximo nominal de $0,5 \mathrm{~mm}$, a un tamaño de tamiz máximo de $1 \mathrm{~mm}$, teniendo como resultado que el porcentaje óptimo de caucho en la mezcla por proceso seco ( $1 \%$ en peso de agregados - $20 \%$ en peso de betún), esto se obtuvo mediante un procedimiento de prueba secuencial en laboratorio, incluyendo: Marshall, sensibilidad a la humedad, tiempo de curado o envejecimiento (Hernández, Witoszek Schultz, Fernández y Moro, 2009).

Por otro lado, Tahami y colaboradores evaluaron el uso de altos contenidos de CR como relleno en mezclas asfálticas para proporcionar un mayor desarrollo mediante el proceso seco considerando el efecto del proceso de curado, el relleno convencional de mezclas se reemplazó con $20 \%$, 40\% y $60 \%$ de CR por peso de rellenoy se evaluaron las propiedades mecánicas de las mezclas modificadas, incluyendo susceptibilidad a la humedad, módulo de rigidez, comportamiento de formación de surcos y vida a fatiga. Los resultados indicaron que la aplicación simultánea de un proceso de curado y CR muy fino en la mezcla asfáltica, mejoró considerablemente la resistencia de las mezclas asfálticas frente a fallas (Tahami, Mirhosseini, Dessouky, Mork y Kavussi, 2019).

Mohamed y colaboradores en su investigación evaluaron las propiedades mecánicas de las mezclas de CR en el asfalto denso graduado fabricadas usando el proceso seco, tuvieron como resultado que las propiedades mecánicas de todas las 
mezclas evaluadas mediante un conjunto de pruebas como la de estabilidad y flujo Marshall, prueba de susceptibilidad a la humedad, ensayo de resistencia a la tracción indirecta, módulo dinámico y número de flujo, mostraron que el uso de CR en el asfalto con 0,75\% del peso del agregado aumentó la estabilidad, el flujo y ha mejorado su resistencia al agrietamiento y deformación permanente (Mohamed, Hassan y Hamdy, 2019).

Hassan y colaboradores realizaron un estudio de laboratorio con el fin de evaluar las propiedades de la mezcla asfáltica modificada con CR utilizando el proceso seco, tuvieron como resultado que el CR mejoró la mayoría de las propiedades de las mezclas asfálticas en comparación con otros tipos de mezclas; esto podría deberse a la interacción parcial entre las partículas de caucho y el betún que actúan simultáneamente como un agregado elástico en la mezcla (Hassan et al., 2019).

\section{Uso del caucho granulado para la mejora de la resistencia y durabilidad de la mezcla asfáltica}

Según los estudios de laboratorio realizados sobre mezclas de asfalto de matriz de piedra (SMA) con fibras naturales y asfalto modificado con $\mathrm{CR}$, se obtuvo que la resistencia a la tracción indirecta, la estabilidad retenida, la resistencia a la susceptibilidad a la humedad, la resistencia a la formación de surcos, la resistencia a la fluencia y la resistencia a la permeabilidad y al envejecimiento mejoran con las mezclas de SMA con CR en comparación con las mezclas de SMA con fibras como estabilizadores (Sharma y Goyal, 2006).

En una investigación realizada por Gallego y colaboradores indicaron que la adición del CR a una mezcla de asfalto graduada mejora la vida útil de las mezclas a la fatiga, la sensibilidad térmica de la mezcla disminuye a medida que aumenta el contenido de CR, mediante el proceso húmedo (Gallego, Castro, Prieto y Vassallo, 2007).

Un estudio realizado por Yang y colaboradores evaluó el desempeño ambiental y mecánico del asfalto de mezcla caliente modificado CR y con Evotherm de tercera generación. Se evaluó el desempeño ambiental que incluyó el ahorro de asfalto modificado con CR, el ahorro de combustible y la reducción de emisiones peligrosas. Por su parte, el desempeño mecánico evaluado incluyó resistencia a la tracción y susceptibilidad a la humedad, resistencia a la formación de surcos, desempeño anti-decapado, desempeño a la fatiga y desempeño a baja temperatura (Yang. et al., 2017).
El estudio de Yang y otros encontró que el ahorro de asfalto con CR fue de 5,8\% en comparación con el asfalto sin modificar, el ahorro de combustible fue de alrededor del 13\% y también se observó una reducción visible de las emisiones peligrosas; asimismo los resultados de rendimiento mecánico mostraron que el asfalto con CR y Evotherm tenía una mejor resistencia a la formación de surcos, un rendimiento a baja temperatura y un mejor rendimiento a la fatiga y resistencia al daño por humedad en comparación con el asfalto sin CR (Yang. et al., 2017).

El estado de la superficie de la carretera es un factor crítico que determina la seguridad vial, la cual debe tener suficiente resistencia al deslizamiento, para ello se desarrolló un estudio en el cual se analizó la resistencia al deslizamiento y el efecto de agregar CR a la mezcla asfáltica, esto se llevó a cabo mediante el proceso seco, se agregó CR a la mezcla asfáltica en proporciones de $0,5 \%, 1 \%, 1,5 \%$ y $2 \%$, teniendo como resultado que la adición de $1 \%$ CR ha mostrado los mejores resultados y proporciona una mejor resistencia al deslizamiento (Ariyapijati, Hadiwardoyo, y Sumabrata, 2019).

Así mismo, Franesqui y colaboradores realizaron una investigación con el objetivo principal de reutilizar el CR, con el fin de reducir el impacto ambiental y alargar la durabilidad de los pavimentos. Mediante estudios experimentales demostraron que estas mezclas con CR mejoran significativamente la resistencia a la formación de surcos, la resistencia al daño por humedad y la estabilidad, cumpliendo así con las especificaciones estándar para pavimentos y ofreciendo suficiente módulo de rigidez dinámica (Franesqui, Yepes y García, 2019).

Liang y colaboradores investigaron un nuevo tipo de mezcla con CR de alta graduación que contiene un 5\% y un $10 \%$ del modificador de CR, el asfalto modificado y no modificado con CR, los cuales se compararon con el mismo grado de rendimiento del asfalto para cumplir con el requisito climático específico, teniendo como resultados de las pruebas de laboratorio donde indicaron que la mezcla con CR de grado denso tenía una vida útil prolongada a la fatiga, mayor resistencia a la fractura y mejor resistencia al agrietamiento térmico que la mezcla sin modificar (Liang, Harvey, Wu, Jiao y Jones, 2020).

Abdulrahman y colaboradores afirman que el asfalto modificado con CR mejora la resistencia a la fatiga, la temperatura y estabilidad frente a la humedad del pavimento flexible, aumentando así su resistencia al surco, también reduce el agrietamiento asociado a baja temperatura del aglomerante mediante la absorción de tensiones. Además, el CR proporciona 
una mejor cohesión y adherencia del betún con el agregado y mejora la recuperación elástica del ligante asfáltico. En conclusión, la superficie de la carretera se vuelve más duradera (Abdulrahman, Hainin, Idham, Hassan y Al, 2020).

Khaled y colaboradores realizaron un estudio con el objetivo de determinar el efecto de la mezcla con CR sobre el rendimiento y las propiedades mecánicas de la mezcla asfáltica en caliente, los resultados de la investigación mostraron que la adición de CR al aglutinante de asfalto en la mezcla fue útil para aumentar la resistencia al daño por humedad, mejorando el módulo resiliente y mejora el potencial para resistir la deformación permanente que se va a presentar con cualquier nivel de carga vehicular, se acelera su manifestación con el aumento de la carga de tráfico (Khaled, Aboud y Al-Hamd, 2020).

\section{Tamaño óptimo del caucho granulado utilizado en las mezclas asfálticas}

Cao y Bai (2008) mencionan en su investigación, que el tamaño óptimo del CR es del tamaño de malla \#20 asegurando que puede dar un mejor asfalto modificado con CR a diferencia del CR de malla \#40 y \#60. Por otro lado, Yu y colaboradores mencionan que para garantizar la coherencia del asfalto modificado con CR, el tamaño óptimo es la malla \#40 (0,425 mm) (Yu, Leng y Wei, 2014), asimismo Pouranian y colaboradores afirman que el tamaño óptimo de CR es de la malla \#40 (Pouranian, Notani, Tabesh, Nazeri y Shishehbor, 2019).

En su investigación realizada, Yildirim y Karacasu (2019) mencionan que el tamaño de las particulas de CR utilizado fue de malla \#30 (0,600 mm) con el propósito de lograr su objetivo de investigar el efecto de la dosis de CR sobre las propiedades del aglutinante de asfalto.

Candra y Siswanto (2019) investigaron el efecto de agregar CR en diferentes porcentajes $(0 \%, 0,5 \%, 1 \%, 1,5 \%, 3 \%, 4,5 \%$ y $6 \%$ del peso agregado) a la mezcla, con tamaños de mallas \#50 y \#100. Demostraron que la mejor adición de CR es del tamaño de partículas de la malla \#100, con un porcentaje de $1 \%$ proporciona un mejor rendimiento que el \#50, según los valores mostrados en la prueba de estabilidad de Marshall.

El CR empleado en el pavimento asfáltico generalmente tiene una cierta finura obtenida triturando neumáticos de desecho de automóviles, es por esa razón que, para la elaboración de su investigación, se selecciona polvo de CR con un tamaño de malla de \#60 como materia prima para la preparación de asfalto modificado compuesto (Li, 2019).

\section{Métodos utilizados en las mezclas asfálticas con caucho granulado}

Se utiliza el método Marshall para seleccionar un contenido óptimo de asfalto para una mejor estabilidad (Aisien, Hymore y Ebewele, 2006), asimismo se aplica para evaluar las características de rendimiento de las mezclas en caliente (HMA). El método se lleva a cabo en un total de tres etapas que incluyen preparación, compresión y ruptura de las muestras, permitiendo determinar los valores de vacíos en la mezcla (Yildirim y Karacasu, 2019). Por otro lado, Yu y colaboradores mencionan que la estabilidad de Marshall es un indicador del rendimiento de la mezcla a alta temperatura (Yu et al., 2019).

El método de resistencia a la tracción indirecta (IDT) modificado, es un método para medir la resistencia a la tracción que también permite determinar la energía hasta la falla y la energía total de fractura, se aplicó usando tres réplicas por mezcla, tanto a mezclas convencionales como mezclas con CR (Zborowski y Kaloush, 2007).

El método de prueba de surco se lleva a cabo mediante un movimiento relativo alternativo con ruedas de carro, lo que hace que la muestra de prueba produzca densificación, cizallamiento y desplazamiento bajo el efecto de repetición de las ruedas, puede simular la verdad física de la rueda del carro que corre bien en el pavimento y la operación es simple con resultados de prueba visuales (Yang, 2014).

La metodología de evaluación del ciclo de vida se utiliza para evaluar y comparar el desempeño ambiental relacionado con tres cursos de desgaste del pavimento de carreteras: mezclas de grado abierto, de grado de separación y de grado denso (Caoa, Leng, Yu y Hsu, 2019).

El método de viscosidad de flujo de corte estable se emplea para que las viscosidades de muchos aglutinantes asfálticos modificados alcancen un estado estacionario a un esfuerzo cortante alto, alrededor de $500 \mathrm{~Pa}$ (Wen y Wang, 2019).

El método básico para evaluar el envejecimiento prolongado en horno se realiza tomando ejemplos de pavimento flexible en campo que presenta buen desempeño, aproximadamente después de 5 años de servicio que ha experimentado envejecimiento prolongado (Negoro, Setyawan y Pramesti, 2020). 


\section{Rendimiento del caucho granulado según el tipo de asfalto}

En su estudio, Rodríguez y colaboradores utilizaron un asfalto B 50/70 (50/70 10mm de penetración), que se utiliza ampliamente para producir mezclas asfálticas a temperaturas normales (Rodríguez, Gallego, Pérez, Bonati y Giuliani, 2014). Se añadió un veinte por ciento en peso de caucho al B 50/70 para obtener el asfalto modificado con $\mathrm{CR}$, el cual dio como resultado que las mezclas asfálticas preparadas con $20 \%$ de aglutinantes con CR se utilizan a menudo cuando se requiere resistencia al agrietamiento. Asimismo, Lopes y colaboradores realizaron su investigación con un asfalto AC 50/70 con caucho reaccionado y activado, obteniendo como resultado un aumento en grado de rendimiento positivo, en punto de reblandecimiento, viscosidad rotacional y densidad, y redujo la penetración (Lopes, Muniz y Rodrigues, 2020).

Bilema y colaboradores utilizaron un asfalto de grado de penetración $80 / 100$, con CR de malla \#20 $(0.15 \mathrm{~mm})$ en diferentes porcentajes, obtuvieron como resultado de la prueba de penetración que a medida que aumentaban los porcentajes de CR, el asfalto tenía más dureza y rigidez debido a valores de penetración más bajos (Bilema, Aman y Ahmad, 2018).

Wang y otros realizaron una investigación con asfalto base de grado de penetración 70/100 comúnmente utilizado en los Países Bajos, con CR de tamaños de partículas que varían de 0 a $0,5 \mathrm{~mm}$, teniendo como resultado que la interacción asfaltocaucho depende en gran medida de la temperatura de mezcla y del tiempo de mezclado (Wang et al., 2018). Asimismo, Wang y colaboradores utilizaron un asfalto de penetración 70/100 como asfalto base para la modificación (Wang, Liu, Erkens y Skarpas, 2020), teniendo como resultado que con un mayor contenido de caucho tiene un espectro de relajación más amplio, lo que significa una transición más gradual del comportamiento elástico al comportamiento viscoso, teniendo en cuenta que una transición elástica-viscosa gradual es beneficiosa para la estabilidad del sistema cuando se encuentran cambios de temperatura.

Bakheit y Xiaoming (2019) utilizaron en su estudio un asfalto de grado de penetración 60/70, con diferentes adiciones de $\mathrm{CR}$, mediante el proceso seco, obteniendo como resultado que la utilización del método seco, podría ser alentador para aumentar la vida útil del pavimento debido a las condiciones de alto tráfico. Por otro lado, Pirmohammad y Khanpour (2020) utilizaron en su investigación un asfalto grado de penetración de 60/70, que se emplea ampliamente en los sistemas de carreteras de Irán, adicionando el 15\% de CR. Afirmaron que el CR mejoró significativamente la resistencia a la fractura del asfalto debido a que el aglutinante de base se volvió dúctil y aumentó la adherencia entre el aglutinante y los agregados.

\section{CONCLUSIONES}

La revisión literaria realizada sugiere que, al adicionar $\mathrm{CR}$ al asfalto, al agregado y a la mezcla, hay una mejora en las propiedades de la mezcla en cuanto a la resistencia a la tracción indirecta, la estabilidad retenida, la resistencia a la susceptibilidad a la humedad, la resistencia a la formación de surcos, la resistencia a la fluencia y la resistencia a la permeabilidad y al envejecimiento, aumenta la vida útil de las mezclas a la fatiga y disminuye el ruido.

En cuanto al porcentaje óptimo de $\mathrm{CR}$ en las mezclas asfálticas varían de acuerdo con la forma como se añade el $\mathrm{CR}$ a las mezclas asfálticas, cuando es agregado al asfalto es recomendado reemplazar un $20 \%$ del peso del asfalto, cuando se agrega al agregado usar $1 \%$ a $3 \%$ del peso total del agregado y cuando se agrega a la mezcla usar $1 \%$ del total de la mezcla, asegurando una resistencia y vida útil duradera.

Según los estudios analizados, es posible afirmar que existen dos procesos para incluir el CR en las mezclas asfálticas, el proceso húmedo y el seco.

El proceso húmedo, el $\mathrm{CR}$ se agrega al asfalto, donde el porcentaje más utilizado se encuentra entre un $14 \%$ y el $20 \%$ del peso total de la mezcla asfalto-caucho y tamaño recomendado es tamiz \#60. En el proceso húmedo se debe de tener en cuenta varios factores concluyentes en el resultado esperado de esta mezcla asfalto-caucho, dichos factores son: el tamaño, la textura y la proporción del RC, el tipo del cemento asfáltico, el tiempo y la temperatura de mezclado y el uso de otros aditivos. El proceso húmedo mejora la propiedad mecánica de resistencia a la formación de surcos.

El proceso seco se genera cuando existe una mezcla directa entre el CR con el agregado antes de agregar el ligante al mezclador de tal manera que el CR es usado como agregado en la mezcla asfáltica, los cuales pueden sumarse como parte del agregado fino y este representa entre el $1 \%$ a $3 \%$ del peso de los agregados. En este proceso para su aplicación no se necesita ningún equipo especial para poder realizar la mezcla ya que el CR entra a mezclarse con los agregados de manera directa. El proceso seco mejora las propiedades mecánicas de resistencia al agrietamiento, deformación permanente y mejora la adherencia entre el aglutinante y los agregados.

Es importante recordar que los resultados obtenidos y los beneficios que se hallaron tras el uso del CR, están ligados al objetivo de cada estudio analizado. 


\section{REFERENCIAS}

Abdulrahman, S., Hainin, M., Idham, M., Hassan, N., y Al, Z. (2020). Review on the potentials of natural rubber in bitumen modification. Earth and Environmental Science, 476(012067), 1-12. doi:10.1088/1755-1315/476/1/012067

Ahmad, M., Beddu, S., Hussain, S., Manan, A., y Itam, Z. (2019). Mechanical properties of hot-mix asphalt using waste crumber rubber and phenol formaldehyde polymer. Materials Science, 6(6), 1164-1175. doi:10.3934/matersci.2019.6.1164

Aisien, F., Hymore, F., y Ebewele, R. (2006). Application of ground scrap tyre rubbers in asphalt concrete pavements. Indian Journal of Engineering y Materials Sciences, 13(4), 333-338.

Al-Salih, A.-S. W. (2020). Using Crumb Rubber to Improve the Bituminous Mixes: Experimental Investigation of Rutting Behavior of Flexible Asphalt Mix for Road Construction. International Conference on Rheology and Modeling of Materials, 1527(1), 1-9. doi:10.1088 / 1742-6596 / 1527/1/012015

Ariyapijati, R., Hadiwardoyo, S., y Sumabrata, R. (2019). Skid resistance of gap graded hot-mix asphalt with added crumb rubber. Journal of Physics: Conference Series, 1376(012010), 1-7. doi:10.1088/1742-6596/1376/1/012010

Bakheit, I., y Xiaoming, H. (2019). Modification of the dry method for mixing crumb rubber modifier with aggregate and asphalt based on the binder mix design. Construction and Building Materials, 220(6), 278-284. doi:10.1016/j.conbuildmat.2019.06.050

Bilema, M., Aman, M., y Ahmad, K. (2018). Investigating the Rheological and Physical Properties for Unaged of Crumb RubberModified Binders Containing Warm Mix Asphalt Additive. Lecture Notes in Civil Engineering, 9(1), $1389-1400$. doi:10.1007/978-981-10-8016-6_100

Bressette, T., Zhou, H., Stonex, A., y Hicks, G. (2008). Asphalt Rubber and Its Potential Use in China. Lui, R., Yang, D y Lu, J. (Editores). En Plan, Build, and Manage Transportation Infrastructure in China, 776-785. doi:10.1061/40952(317)75

Candra, P., y Siswanto, H. (2019). Marshall Characteristics of Asphalt Concrete Wearing Course Using Crumb Rubber Modified of Motorcycle Tire Waste As Additive. Materials Science Forum, 961, 57-61. doi:10.4028/www.scientific.net/MSF.961.57

Cao, W. (2007). Study on properties of recycled tire rubber modified asphalt mixtures using dry process. Construction and Building Materials, 21(5), 1011-1015. doi:10.1016/j.conbuildmat.2006.02.004

Cao, R., y Bai, Q. (2008). Laboratory Evaluation of Performances of Asphalt Ruber and Gap Graded Mixtures. Plan, Build, And Manage Transportation Infrastructure In China, 317, 786-798. doi:10.1061/40952(317)76

Caoa, R., Leng, Z., Yu, H., y Hsu, S. C. (2019). Comparative life cycle assessment of warm mix technologies in asphalt rubber pavements with uncertainty analysis. Resources, Conservation E Recycling, 147, 137-144. doi:10.1016/j.resconrec.2019.04.031

Dantas, S., Farias, M., Pais, J., y Pereira, P. (2006). Dense Graded Hot Mixes using Asphalt-Rubber Binders with High Rubber Contents. Road Materials and Pavement Design, 7(1), 29-46. doi:10.1080/14680629.2006.9690025

Dong, Y., y Tan, Y. (2011). Mix Design and Performance of Crumb Rubber Modified Asphalt SMA. Geotechnical Special Publication, 212, 78-86. doi:10.1061/47623(402)10

Franesqui, M., Yepes, J., y García, C. (2019). Improvement of moisture damage resistance and permanent deformation performance of asphalt mixtures with marginal porous volcanic aggregates using crumb rubber modified bitumen. Construction and Building Materials, 201, 328-339. doi:10.1016/j.conbuildmat.2018.12.181

Gallego, J., Castro, M., Prieto, J., y Vassallo, J. (2007). Thermal Sensitivity and Fatigue Life of Gap-Graded Asphalt Mixes Incorporating Crumb Rubber from Tire Waste. Journal of the Transportation Research Board, 1998, 132-139. doi:10.3141/1998-16

Gong, J., Liu, Y., Wang, Q., Xi, Z., Cai, J., Ding, G., y Xie, H. (2019). Performance evaluation of warm mix asphalt additive modified epoxy asphalt rubbers. Construction and Building Materials, 204, 288-295. doi:10.1016/j.conbuildmat.2019.01.197

Hamzani, Munirwansyah, Hasan, y Sugiarto. (2019). The influence of the using waste tire rubber and natural ziolite as Asphalt and Cement replacements to compressive strength of Semi-Flexible Pavement. Materials Science and Engineering, 523(1), 1-7. doi:10.1088/1757-899X/523/1/012037 
Hassan, N., Almusawi, A., Mahmud, M., Abdullah, A., Mohd, N., Mashros, N., Putra, R., y Yusoff, N. (2019). Engineering properties of crumb rubber modified dense-graded asphalt mixtures using dry process. Earth and Environmental Science, 220(1), 1-11. doi:10.1088/1755-1315/220/1/012009

Hernández Olivares, F., Witoszek Schultz, B., Fernández, M., y Moro, C. (2009). Rubber-modified hot-mix asphalt pavement by dry process. International Journal of Pavement Engineering, 10(4), 277-288. doi:10.1080/10298430802169416

Kartika, L., Hadiwardoyo, S., y Sumabrata, R. (2019). Rutting Deformation of Gap-Graded Hot-Mix Asphalt with Added of Waste Tire Rubber. Process and Design for Sustainable Urban Development, 2114(4), 1-8. doi:10.1063/1.5112442

Khaled, T., Aboud, G., y Al-Hamd, R. (2020). Study the effect of adding crumb rubber on the performance of hot mix asphalt. Materials science and engineering, 737(1), 1-12. doi:10.1088/1757-899X/737/1/012129

Khalili, M., Jadidi, K., Karakouzian, M., y Amirkhanian, S. (2019). Rheological properties of modified crumb rubber asphalt binder and selecting the best modified binder using AHP method. Case Studies in Construction Materials, 11, 1-20. doi:10.1016/j. cscm.2019.e00276

Kim, H. S., Lee, S. J., y Amirkhanian, S. (2010). Rheology Investigation of Crumb Rubber Modified Asphalt Binders. Journal of Civil Engineering, 14(6), 839-843. doi:10.1007/s12205-010-1020-9

Li, Y. (2019). Study on SBS/rubber composite modified asphalt ultra-thin wear layer. Materials Science and Engineering, 612(2), 1-8. doi:10.1088/1757-899X/612/2/022067

Liang, Y., Harvey, J., Wu, R., Jiao, L., y Jones, D. (2020). Fatigue and Fracture Properties of Asphalt Mixes Containing Low Content of Crumb-Rubber Modifier. Journal of Materials in Civil Engineering, 32(10), 1-9. doi:10.1061/(ASCE)MT.1943-5533.0003371

Lo Presti, D. (2013). Recycled Tyre Rubber Modified Bitumens for road asphalt mixtures: A literature review. Construction and Building Materials, 49, 863-881. doi:10.1016/j.conbuildmat.2013.09.007

Lopes, L., Muniz, M., y Rodrigues, L. (2020). Fatigue tests and damage analyses in modified binders and gap-graded asphalt mixtures with Reacted and Activated Rubber - RAR. Road Materials and Pavement Design, 21(1), 1-22. doi:10.1080/14680629.20 19.1710553

Ma, T., Wang, H., Zhao, Y., Huang, X., y Wang, S. (2016). Laboratory Investigation of Crumb Rubber Modified Asphalt Binder and Mixtures with Warm-Mix Additives. International Journal of Civil Engineering, 15(2), 1-10. doi:10.1007 / s40999-0160040-3

Mohamed, S., Hassan, M., y Hamdy, I. (2019). The Potential use of Crumb Rubber in Hot Asphalt Mixes in Egypt using Dry Process. International Journal of Engineering and Advanced Technology (IJEAT), 9(1), 4356-4360. doi:10.35940 / ijeat. A1952.109119

Munir, M., Elnour, A., Huda, S., y Zeiada, W. (2020). Activated Crumb Rubber Modified Binder as a Sustainable Paving Material: Pavement Performance Consideration. Advances in Science and Engineering Technology International Conferences, 1-7. doi:10.1109/ASET48392.2020.9118219

Negoro, M., Setyawan, A., y Pramesti, F. (2020). The Aging Properties Of Thin Surfacing Hot Mix Asphalt Containing Crumb Rubber As Aggregate Replacement. International Journal of Scientific E Technology Research, 9(4), 880-884.

Pirmohammad, S., y Khanpour, M. (2020). Fracture strength of warm mix asphalt concretes modified with crumb rubber subjected to variable temperatures. Road Materials and Pavement Design, 21(1), 1-20. doi:10.1080/14680629.2020.1724819

Pouranian, M., Notani, M., Tabesh, M., Nazeri, B., y Shishehbor, M. (2019). Rheological and environmental characteristics of crumb rubber asphalt binders containing non-foaming warm mix asphalt additives. Construction and Building Materials, 238, 1-17. doi:10.1016/j.conbuildmat.2019.117707

Rodríguez, A., Gallego, J., Pérez, I., Bonati, A., y Giuliani, F. (2014). High and low temperature properties of crumb rubber modified binders containing warm mix asphalt additives. Construction and Building Materials, 53, 460-466. doi:10.1016/j. conbuildmat.2013.12.026 
Rodríguez, I., Tarpoudi, F., Cavalli, M., Poulikakos, L., y Bueno, M. (2020). Microstructure analysis and mechanical performance of crumb rubber. Construction and Building Materials, 259, 119662-119671. doi:10.1016/j.conbuildmat.2020.119662

Saeed, M., Aman, M., Ahmad, K., Yero, T., y Chinade, A. (2018). Effect of Crumb Rubber Modifier on the Fatigue Performance of Warm Mix Asphalt. Lecture Notes in Civil Engineering, 9, 1367-1376. doi:10.1007/978-981-10-8016-6_98

Sharma, V., y Goyal, S. (2006). Comparative study of performance of natural fibres and crumb rubber modified stone matrix asphalt mixtures. Canadian Journal of Civil Engineering, 33(2), 134-139. doi:10.1139/L05-096

Shen, J., Amirkhanian, S., Lee, S., y Putman, B. (2006). Recycling of Laboratory-Prepared Reclaimed Asphalt Pavement Mixtures Containing Crumb Rubber-Modified Binders in Hot-Mix Asphalt. Journal of the Transportation Research Board, 1962, 71-78. doi:10.3141/1962-09

Tahami, S., Mirhosseini, A., Dessouky, S., Mork, H., y Kavussi, A. (2019). The use of high content of fine crumb rubber in asphalt mixes using dry process. Construction and Building Materials, 222, 643-653. doi:10.1016/j.conbuildmat.2019.06.180

Wang, J., y Zeng, X. (2006). Influence of Temperature and Pressure on the Dynamic Properties of Rubber-Modified Asphalt Concrete. Journal of Materials in Civil Engineering, 18(1), 125-131. doi:10.1061/ASCE 0899-1561 2006 18: 1125

Wang, H., Liu, X., Zhang, H., Apostolidis, P., Scarpas, T., y Erkens, S. (2018). Asphalt-rubber interaction and performance evaluation of rubberised asphalt binders containing non-foaming warm-mix additives. Road Materials and Pavement Design, 21(6), 1-23. doi:10.1080/14680629.2018.1561380

Wang, H., Liu, X., Erkens, S., y Skarpas, A. (2020). Experimental characterization of storage stability of crumb rubber modified bitumen with warm-mix additives. Construction and Building Materials, 249, 1-10. doi:10.1016/j.conbuildmat.2020.118840

Wen, Y., y Wang, Y. (2019). High-Temperature Rheological Properties of Asphalt Binders with Polymeric, Warm-Mix, and Rubber Particulate Additives. American Society of Civil Engineers, 31(3), 1-16. doi:10.1061/(ASCE)MT.1943-5533.0002589

Yang, Y. (2014). Research on Ice-Broken Effect of Granulated Rubber Asphalt Mixture High-elastic Deck Pavement on Bridge. Applied Mechanics and Materials, 587, 971-975. doi:10.4028/www.scientific.net/AMM.587-589.971

Yang, X., You, Z., Rosli, M., Diab, A., Shao, H., Chen, S., y Ge, D. (2017). Environmental and Mechanical Performance of Crumb Rubber Modified Warm Mix Asphalt using Evotherm. Journal of Cleaner Production, 159, 1-34. doi:10.1016/j.jclepro.2017.04.168

Yildirim, Z., y Karacasu, M. (2019). Modelling of waste rubber and glass fibber with response surface method in hot mix asphalt. Construction and Building Materials, 227, 117070-117083. doi:10.1016/j.conbuildmat.2019.117070

Yu, X., Leng, Z., y Wei, T. (2014). Investigation of the Rheological Modification Mechanism of Warm-mix Additives on Crumb Rubber Modified Asphalt. Journal of Materials in Civil Engineering, 26(2), 1-40. doi:10.1061/(ASCE)MT.1943-5533.0000808

Yu, H., Zhu, Z., Leng, Z., Wu, C., Zhang, Z., y Wang, D. (2019). Effect of Mixing Sequence on Asphalt Mixtures Containing Waste Tire Rubber and Warm Mix Surfactants. Journal of Cleaner Production, 246, 1-39. doi:10.1016/j.jclepro.2019.119008

Zborowski, A., y Kaloush, K. (2007). Predictive Equations to Evaluate Thermal Fracture of Asphalt Rubber Mixtures. Road Materials and Pavement Design, 8(4), 819-833. doi: 10.1080/14680629.2007.9690101 